J. Lake Sci.(湖泊科学), 2019, 31(3): 881-890

DOI 10. 18307/2019. 0326

(C) 2019 by Journal of Lake Sciences

\title{
水位变化对干涸湖底沉积物有机碳矿化的影响
}

\author{
李典鹏 ${ }^{1}$, 姚美思 ${ }^{1}$, 孙 涛 $^{1}$, 刘隋贺是 ${ }^{1}$, 张 凯 $^{1,2}$, 贾宏涛 ${ }^{1,2 * *}$ \\ $(1:$ 新疆农业大学草业与环境科学学院, 乌鲁木齐 830052) \\ $(2:$ 新疆土壤与植物生态过程重点实验室, 乌鲁木齐 830052)
}

\begin{abstract}
摘 要: 人为干扰和气候变化会改变湖泊水位状态, 明确不同水位条件下湖泊沉积物有机碳矿化特征及其影响因素, 对 了解内陆水生态系统碳循环具有重要意义. 为探究干旱区典型盐湖沉积物有机碳矿化速率对水位变化的响应, 以巴里坤 湖干涸湖底原状沉积物为研究对象, 初步探究了 $0(\mathrm{~T} 1) 、-9(\mathrm{~T} 2) 、-23$ ( T3) 、-34 (T4) 和 $-45 \mathrm{~cm}$ ( T5) 水位处理对沉积 物有机碳矿化速率的影响. 结果表明, T1、T2 和 T3 处理有机碳矿化速率在试验初期较高 $(0 \sim 10 \mathrm{~d}), 10 \mathrm{~d}$ 后缓慢下降, T4 和 T5 处理有机碳矿化速率呈先增加后降低趋势; T1 $\left(1.718 \mu \mathrm{mol} /\left(\mathrm{m}^{2} \cdot \mathrm{s}\right)\right)$ 与 T3 $\left(1.784 \mu \mathrm{mol} /\left(\mathrm{m}^{2} \cdot \mathrm{s}\right)\right)$ 处理有机碳矿化 速率不存在显著差异, T1 处理有机碳矿化速率是 T2、T4 和 T5 处理的 1.09、3.31 和 3.57 倍, 不同处理有机碳累积矿化量 表现为 $\mathrm{T} 3>\mathrm{T} 1>\mathrm{T} 2>\mathrm{T} 4>\mathrm{T} 5$. 有机碳累积矿化量 $\left(C_{t}\right)$ 占沉积物有机碳 $\left(C_{0}\right)$ 的比例 $\left(\mathrm{C}_{t} / \mathrm{C}_{0}\right)$ 介于 $0.012 \sim 0.044$ 之间, 沉积物 有机碳潜在排放量 $\left(C_{i}\right)$ 占 $C_{0}$ 的比例 $\left(C_{i} / C_{0}\right)$ 介于 $0.018 \sim 0.045$ 之间; 水位降低, 沉积物有机碳矿化常数 ( $k$ 值) 减小, T1 处理 $k$ 值最大 $(0.137 \mathrm{~d}), \mathrm{T} 4$ 处理最小 $(0.032 \mathrm{~d})$. 线性方程 $C_{\mathrm{r}}=0.008 x+0.488$ 能较好地模拟有机碳矿化速率 $\left(C_{\mathrm{r}}\right)$ 与水位 $(x)$ 的关系; 不同水位处理有机碳矿化速率与模拟柱中沉积物 $5 \mathrm{~cm}$ 温度呈显著的指数函数关系, T4、T5 处理有机碳矿化 温度敏感系数 $\left(Q_{10}\right)$ 显著高于 $\mathrm{T} 1 、 \mathrm{~T} 2$ 和 $\mathrm{T} 3$ 处理, 即水位降低增加了巴里坤湖干涸湖底沉积物 $\mathrm{Q}_{10}$. 因此, 就巴里坤湖干 涸湖底沉积物而言, 水位从 $0 \mathrm{~cm}$ 降至 $-45 \mathrm{~cm}$ 时有机碳矿化速率降低, $Q_{10}$ 增加; 反之水位上升则会促进有机碳矿化分 解, $Q_{10}$ 降低. 水位持续下降抑制有机碳矿化可能是维持干旱区盐湖沉积物碳库稳定的机制之一.

关键词: 干旱区盐湖;水位梯度;碳排放;湖底沉积物;温度敏感系数;巴里坤湖
\end{abstract}

\section{Effect of different water level gradients on the mineralization of sediment organic carbon in a dry lake}

\author{
LI Dianpeng ${ }^{1}$, YAO Meisi ${ }^{1}$, SUN Tao ${ }^{1}$, LIU Suiyunhao ${ }^{1}$, ZHANG Kai ${ }^{1,2} \&$ JIA Hongtao ${ }^{1,2 * *}$ \\ (1: College of Grassland and Environmental Science, Xinjiang Agricultural University, Urumqi 830052, P.R.China) \\ (2: Xinjiang Key Laboratory of Soil and Plant Ecological Processes, Urumqi 830052, P.R. China)
}

Abstract: : The loss of organic carbon during passage through the continuum of inland waters from soils to the sea is a critical issue for the global carbon cycle. However, the amount of organic carbon mineralized and released to the atmosphere during its transport remains an open question, hampered by the absence of predictors of organic carbon mineralization rates. The lake water level can be affected by human disturbance and climate change, and thus change the mineralization of the sediment organic carbon. The primary objective of this study was to study the influence of different water level gradients on the mineralization of sediment organic carbon in a saline lake in arid region. Sediments at 0-50 cm depth were sampled from Lake Barkol. The mineralization rates of sediment organic carbon were measured by Li-COR 8100A under five underground water level treatments ( T1, T2, T3, T4 and T5 represent underground water level $0,-9,-23,-34$ and $-45 \mathrm{~cm}$ ) were settled and sediment organic carbon mineralization rates were measured by Li-COR $8100 \mathrm{~A}$. Results showed the mineralization rates of sediment organic carbon under T1, T2 and T3 treatments were higher $(0-10 \mathrm{~d})$ at the beginning of the experiment, and then decreased slowly. The carbon mineralization rates under T4 and T5 treatments increased firstly and then decreased. The mineralization rate of organic carbon under T1 treatment was 1.09 , 3.31 and 3.57 times higher than that under T2, T4 and T5 treatments, respectively. The cumulative mineralization of sediment organic car-

* 国家自然科学基金项目 (31560171) 和新疆农业大学研究生科研创新项目 (XJAUGRI2018011) 联合资助. 2018-1023 收稿;2018-11-16 收修改稿. 李典鹏(1992 ), 男, 硕士研究生; E-mail: 1ldp05120@ 126.com.

** 通信作者;E-mail: hongtaojia@ 126.com. 
bon under different treatments is $\mathrm{T} 3>\mathrm{T} 1>\mathrm{T} 2>\mathrm{T} 4>\mathrm{T} 5$. The ratios of cumulative mineralization of organic carbon $\left(C_{t}\right)$ to total sediment organic carbon $\left(C_{0}\right)\left(C_{t} / C_{0}\right)$ are ranged from $1.2 \%$ to $4.4 \%$, and the ratios of potential organic carbon emissions $\left(C_{i}\right)$ to $C_{0}\left(C_{i} / C_{0}\right)$ are ranged from $1.8 \%$ to $4.5 \%$. The decrease of underground water level reduced the mineralization constant $(k$ value) of sediment organic carbon. The $k$ value under T1 treatment was max $(0.137 \mathrm{~d})$, and that under T4 treatment was lowest $(0.032$ d). The best fitting model explaining the relationship between sediment organic carbon mineralization rate and water level $(x, \mathrm{~cm})$ was $C_{\mathrm{r}}=0.008 x+0.488$. There were significant positive relationships between organic carbon mineralization rate and sediment temperature at $5 \mathrm{~cm}$ depth. Water level had significant effect on the temperature sensitive of sediment organic carbon mineralization $\left(Q_{10}\right)$. The $Q_{10}$ was highest under T5 treatment (2.92), followed by $\mathrm{T} 4$ (2.54), and the T1 treatment had the smallest value (1.92). These results indicated that the decrease of underground water level would reduce the mineralization rate of organic carbon and increas $Q_{10}$. The continuous decline of underground water level inhibits organic carbon mineralization, which may be a mechanism to maintain the stability of carbon pools of lake sediment in arid regions.

Keywords: Arid region saline lake; water level gradient; carbon emission; lake sediment; temperature sensitivity $\left(Q_{10}\right)$; Lake Barkol

自 $1990 \mathrm{~s}$ 以来, 地球表面温度上升了 $0.65 \sim 1.06^{\circ} \mathrm{C}^{[1]}$, 除水气 $\left(\mathrm{H}_{2} \mathrm{O}\right)$ 外, 大气中二氧化碳 $\left(\mathrm{CO}_{2}\right)$ 是主要的 温室气体, 对温室效应的贡献率可达 $60 \%{ }^{[2]}$. 全球内陆水体面积虽仅占陆地总面积的 $3 \%$,但易受人类活动 和气候变化的影响, 其在全球碳循环中所占的比重不可忽视 ${ }^{[3-4]}$. 全球大于 $0.1 \mathrm{~km}^{2}$ 的湖泊面积约为 $3.2 \times 10^{6}$ $\mathrm{km}^{2}$, 占陆地表面积的 $2.4 \%{ }^{[5]}$. 水体沉积物中有机质在微生物的作用下, 将其中的有机碳转变为 $\mathrm{CO}_{2}$ 或 $\mathrm{CH}_{4}$ 等气体, 每年可将 $2.1 \mathrm{Pg}$ 碳排放到大气中, 约占陆地生态系统土壤碳排放的 $3 \%$, 在全球碳循环和区域气候 调节中发挥着重要作用 ${ }^{[6-9]}$. 因此, 水体沉积物有机碳矿化产生的温室气体排放对全球温室效应起正反馈调 节作用.

湖泊生态系统 $\mathrm{CO}_{2}$ 产生是一个生物过程, 非生物因素 $\mathrm{pH}$ 、有机碳质量、温度以及含水量等是影响微生物 矿化有机碳的重要因素 ${ }^{[10]}$. Gudasz 等 ${ }^{[11]}$ 研究表明, 有机碳矿化速率与温度呈极显著正相关; 不同纬度湖泊 对温度变化的响应也不同, 例如, 巴西湖泊沉积物有机碳矿化速率是瑞典湖泊的 2.4 倍 ${ }^{[12]}$; 除表层沉积物外, 深层沉积物有机碳矿化分解也与温度显著相关 ${ }^{[13]}$. 此外, 温度变化引起的沉积物中盐分、总氮和叶绿素 $\mathrm{a}$ 等因 子的变化也是影响有机碳矿化的主要因素 ${ }^{[14]}$. 近年来, 受人类活动和气候变化的影响, 全球主要湖泊特别 是盐湖面积处于萎缩状态 ${ }^{[15]}$, 面积的减小会导致水位降低, 这也会影响有机碳的矿化 ${ }^{[16]}$. 欧强等 ${ }^{[17]}$ 对滨海 湿地的研究表明, 低水位和高水位处理有机碳矿化速率均低于中水位, 碳排放温度敏感系数也随之改变, 而 沿海滩涂有机碳矿化对水位变化的响应与滨海湿地一致 ${ }^{[18]}$. 干旱区巴音布鲁克天鹅湖 $\mathrm{CO}_{2}$ 排放对水位变化 同样敏感, $-20 \mathrm{~cm}$ 水位有机碳矿化速率最高,低水位处理对温度变化的响应最敏感 ${ }^{[19]}$. 综上,水位变化可显 著改变沉积物有机碳矿化速率及温度敏感性. 但干旱区盐湖干涸湖底沉积物有机碳矿化对水位变化的响应 特征仍不明确.

在全球升温背景下, 干旱灾害风险不断加剧, 加之湖区内不合理的开采利用, 导致湖泊水位不断下 降 ${ }^{[15]}$. 巴里坤湖作为干旱区的典型盐湖, 水域面积从 1984 年的 $112.15 \mathrm{~km}^{2}$ 下降至如今不足 $60 \mathrm{~km}^{2}$. 而干涸 湖底面积不断扩大导致水位的降低, 可能会改变沉积物有机碳的矿化规律. 因此, 本研究以巴里坤湖干涸湖 底原状沉积物为研究对象, 通过异位原状沉积物模拟水位梯度变化. 试探究: 1) 巴里坤湖干涸湖底沉积物有 机碳矿化速率对水位变化响应及与水位的相关性;2) 不同水位条件干涸湖底沉积物有机碳累积矿化量及动 力学特征; 3) 明确干涸湖底沉积物有机碳矿化与温度的相关性及温度敏感系数, 以期为全面认识湖泊生态 系统碳循环过程提供基础资料和科学参考.

\section{1 材料与方法}

\section{1 研究区概况}

巴里坤湖 ( $43^{\circ} 36^{\prime} \sim 43^{\circ} 43^{\prime} \mathrm{N}, 92^{\circ} 43^{\prime} \sim 92^{\circ} 51^{\prime} \mathrm{E}$ ) 位于天山东段巴里坤山与莫钦乌拉山之间的巴里坤盆 地, 属典型的封闭型干涸尾问湖, 距离巴里坤哈萨克自治县城西北约 $18.0 \mathrm{~km}$ (图 1a). 巴里坤湖主要的地表 径流补给源自布鲁克山北坡, 另外地下水也对湖泊有一定补给, 补给系数为 38.8 . 湖泊表层卤水密度为 1.172 
$\mathrm{g} / \mathrm{cm}^{3}, \mathrm{pH}$ 值为 7.59 , 矿化度高达 $204.76 \mathrm{~g} / \mathrm{L}$, 属新生代山间构造断陷形成的硫酸美亚型盐湖. 湖区属温带大 陆性干旱气候, 年平均气温为 $1.1^{\circ} \mathrm{C}$, 极端低温 $-43.6^{\circ} \mathrm{C}$, 极端高温 $33.5^{\circ} \mathrm{C}$. 多年平均日照时数 $3211 \mathrm{~h}$, 无霜期 $101 \mathrm{~d}$. 年平均降水量为 $210 \mathrm{~mm}$, 年均蒸发量 $2250 \mathrm{~mm}^{[20]}$.

\section{2 样品采集}

2016 年 6 月,于巴里坤湖干涸湖底区 $\left(43^{\circ} 43^{\prime} 35^{\prime \prime} \mathrm{N}, 92^{\circ} 46^{\prime} 39^{\prime \prime} \mathrm{E}\right.$; 海拔 $1700 \mathrm{~m}$ ) 选取组成、结构、密度等相 对一致且湖岸距离相同的区域采集原状沉积物 (图 1), 采用直径为 $20 \mathrm{~cm}$ 、高为 $60 \mathrm{~cm}$ 的 PVC 管采集原状沉 积物柱, 柱高 $50 \mathrm{~cm}$, 共采集 20 个. 同时采集 $0 \sim 50 \mathrm{~cm}$ 沉积物, 带回实验室进行水位控制试验和基本理化性 质分析. 同时在盐分富集区采集沉积物表面积盐,用于水位控制试验.
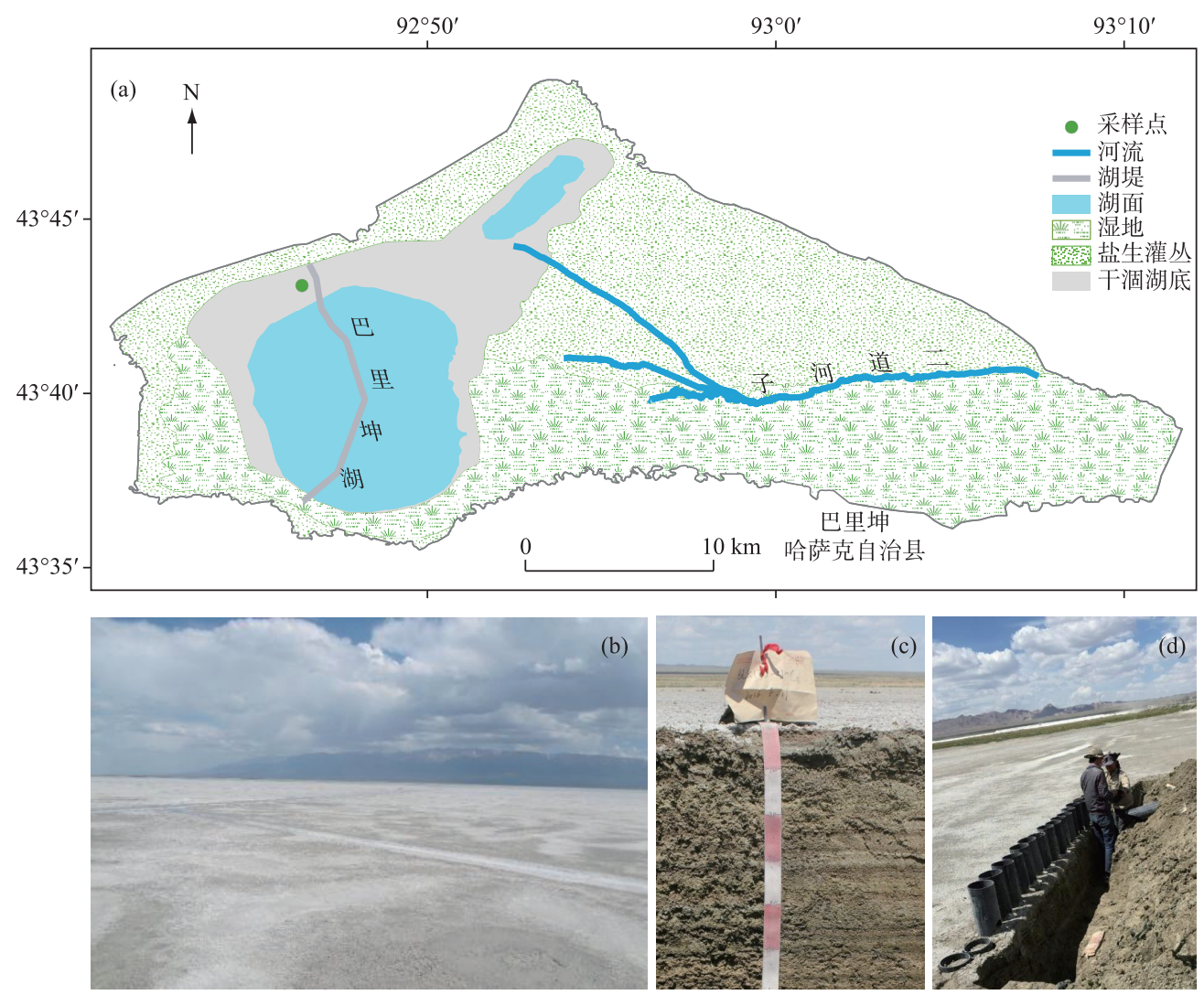

图 1 研究区示意图地貌和沉积物剖面特征:

研究区示意图 (a); 干涸湖底区景观 (b) ; 干涸湖底沉积物剖面 (c); 原状沉积物采集现场 (d)

Fig.1 Location of the study area, topography and sediment profile characteristics

\section{3 试验设计}

开始试验前对沉积物柱底部进行密封, 采用 Li-8100 ( LI-COR, Lincoln, USA) 碳通量测定系统分别测定 了 20 个沉积物柱的基础有机碳矿化速率, 然后根据有机碳基础矿化速率将沉积物柱分为 5 组, 分别记作 $\mathrm{T} 1 、 \mathrm{~T} 2 、 \mathrm{~T} 3 、 \mathrm{~T} 4$ 和 T5, 有机碳矿化速率分别为 $2.35 \pm 0.14 、 2.20 \pm 0.07 、 2.23 \pm 0.15 、 2.33 \pm 0.17$ 和 $2.23 \pm 0.07 \mu \mathrm{mol} /$ $\left(\mathrm{m}^{2} \cdot \mathrm{s}\right), 5$ 组沉积物柱基础有机碳矿化速率不存在显著性差异 $(P>0.05)$.

湖底沉积物是客观存在的历史自然体,可敏感地记录历史时期气候和水文特征. 水位变化对沉积物的 影响主要体现在颜色方面, 例如, 长期水分过饱和, 铁锰化合物在氙气条件下还原, 使沉积物形成灰蓝色至 青灰色层次或具红棕色锈纹、锈斑和铁锰结核层; 水流量大小会对沉积物径级和结构产生较大影响. 谢帕德 和福克碎屑沉积物最常用的分类方法 ${ }^{[21]}$ 仅考虑沉积物粒径大小, 忽略了颜色和孔隙等方面存在的差异, 不 
能较好地反映沉积物特性. 因此, 本研究参照中国土壤发生分类标准对干涸湖底沉积物进行层次划分 (图 1c), 干涸湖底沉积物在 $-9 、-23 、-34$ 和 $-45 \mathrm{~cm}$ 处颜色、粒径、孔隙和盐分上均存在一定差异, 不同层次沉积 物的描述情况见表 1. 故本试验设置了 $0(\mathrm{~T} 1) 、-9(\mathrm{~T} 2) 、-23(\mathrm{~T} 3) 、-34(\mathrm{~T} 4)$ 和 $-45 \mathrm{~cm}(\mathrm{~T} 5)$ 水位处理, 模拟不 同水文特征期的沉积物有机碳矿化速率.

\section{表 1 不同深度沉积物特点}

Tab.1 Properties of sediment at different depths

\begin{tabular}{ll}
\hline 深度/cm & 沉积物特点 \\
\hline $0 \sim 9$ & 褐色,粉质,粒状,结构松散,孔隙小且多,盐分表聚 \\
$9 \sim 23$ & 土黄色,粉质,粒状,结构极疏松,孔隙少较多 \\
$23 \sim 34$ & 浅棕色,粉黏质地,粒状,结构疏松,孔隙较少 \\
$34 \sim 45$ & 浅褐色,黏质,块状,结构坚实,孔隙较少 \\
\hline
\end{tabular}

将用 PVC 管采集的原状沉积物放人 1 连 4 的水位控制装置中 (图 2), 底部和水管连接 处涂抹胶水保证装置良好的密封状态, 置于室 内. 不同水位处理设置 3 个重复,每个水位梯 度另设一个沉积物柱用于监测模拟柱温度 (ST). 2016 年 6 月 24 日进行淹水, 记为试验 第 1 天,当不同处理水位保持稳定后测定有机 碳矿化速率. 监测于每天 11:00 进行, 监测频 率为试验前期 $(0 \sim 10 \mathrm{~d})$ 每 $2 \mathrm{~d}$ 测定一次, 试验 中后期 (10 d 以后) 每 $4 \mathrm{~d}$ 测定一次, 7 月 25 日结束. 为最大限度模拟盐湖水体矿化度, 试验用水与湖水矿化 度基本保持一致,使用巴里坤湖采集的粗盐将蒸馏水矿化度调至约 $204 \mathrm{~g} / \mathrm{L}$, 用于装置的水位调节. 试验期 间内每 $2 \mathrm{~d}$ 观测一次水位高度并补充水分至对应高度. 在测定沉积物有机碳矿化速率的同时,记录沉积物柱 温度和水位控制柱中水温 (WT).

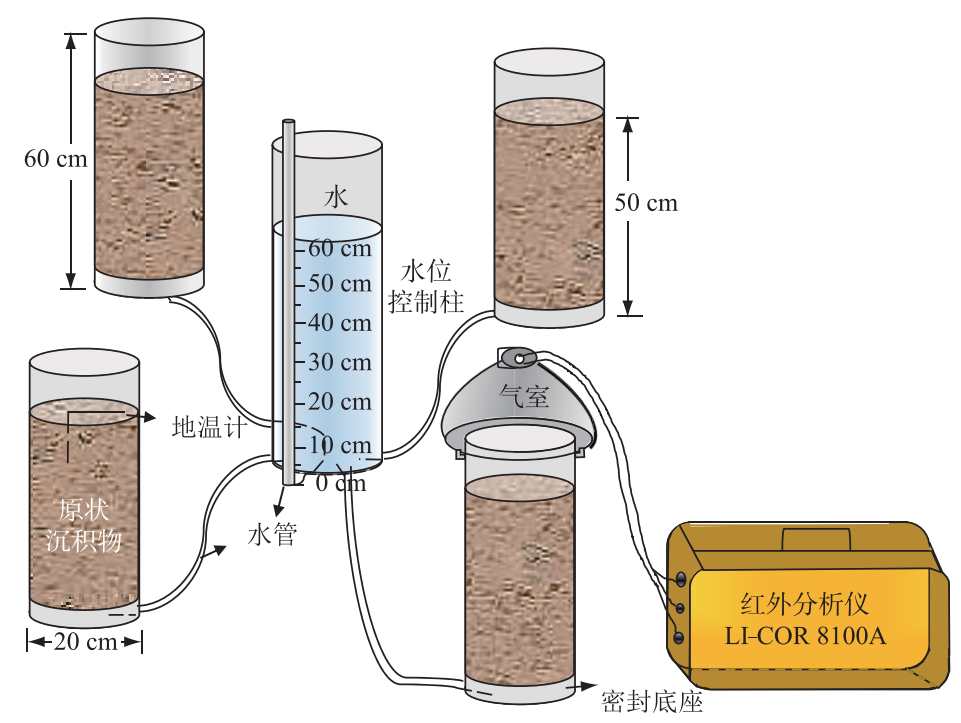

图 2 模拟水位梯度装置示意图

Fig.2 Diagram of water level installation

\section{4 干涸湖底沉积物基本理化性质测定方法}

将采集的 $0 \sim 50 \mathrm{~cm}$ 沉积物经自然风干后充分混匀, 过 $1 \mathrm{~mm}$ 标准篮后进行 $\mathrm{pH}$ 、电导率和盐分离子的测 定, 过 $0.25 \mathrm{~mm}$ 标准篮的沉积物用于有机质的测定. $\mathrm{pH}$ 采用酸度计测定, 电导率采用电导率仪测定, 总有机 碳采用重铬酸钾一硫酸外加热法测定 ${ }^{[22]}$. 用孔径 $0.7 \mu \mathrm{m}$ 的 Whatman GF/F 型玻璃纤维质滤膜过滤水样,然 后用美国戴安 IC-900 型离子色谱仪测定阴离子含量; 用 TAS-990 型原子吸收分光光度计测定阳离子含量. 巴里坤湖干涸湖底沉积物基础理化性质见表 2. 
表 2 干涸湖底沉积物基本性质"

Tab.2 Properties of sediment in Lake Barkol

\begin{tabular}{lcccccccccc}
\hline \multirow{2}{*}{$\begin{array}{c}\text { 有机质/ } \\
(\mathrm{g} / \mathrm{kg})\end{array}$} & $\begin{array}{c}\text { 电导率/ } \\
(\mathrm{mSS} / \mathrm{cm})\end{array}$ & $\mathrm{pH}$ & \multicolumn{9}{c}{ 含量 $/(\mathrm{g} / \mathrm{kg})$} \\
\cline { 4 - 10 } & & $\mathrm{Na}^{+}$ & $\mathrm{K}^{+}$ & $\mathrm{Ca}^{2+}$ & $\mathrm{Mg}^{2+}$ & $\mathrm{Cl}^{-}$ & $\mathrm{SO}_{4}^{2-}$ & $\mathrm{HCO}_{3}^{-}$ & $\mathrm{CO}_{3}^{2-}$ \\
\hline $39.08 \pm 1.07$ & $41.14 \pm 7.92$ & $9.23 \pm 0.17$ & 5.32 & 0.21 & 1.38 & 0.13 & 0.37 & 7.36 & 0.04 & 0.01 \\
\hline
\end{tabular}

* 表中数据为平均值土标准误.

\section{5 数据处理方法}

沉积物 $\mathrm{CO}_{2}$ 释放累积过程符合一级反应动力学方程 ${ }^{[23]}$, 采用方程模型对 $\mathrm{CO}_{2}$ 累积排放量进行拟合, 如:

$$
C_{t}=C_{i}\left(1-\mathrm{e}^{-\mathrm{k} t}\right)
$$

式中, $C_{t}$ 为 $t$ 时间内沉积物 $\mathrm{CO}_{2}$ 累积排放量, $\mathrm{g} / \mathrm{m}^{2} ; C_{i}$ 为沉积物有机碳潜在排放量, $\mathrm{g} / \mathrm{m}^{2} ; \mathrm{k}$ 为有机碳矿化常 数, $\mathrm{d} ; t$ 为培养天数, $\mathrm{d}$.

采用 Vant't Hoff 指数函数拟合有机碳矿化速率与温度的关系 (公式 (2)), 计算沉积物有机碳矿化的温 度敏感性系数 $\left(Q_{10}\right)$, 即温度每增加 $10^{\circ} \mathrm{C}$, 有机碳矿化速率所增加的倍数采用公式 (3) 计算 ${ }^{[18]}$; 采用线性方 程模拟水位与有机碳矿化速率之间的关系(公式(4)):

$$
\begin{aligned}
& C_{\mathrm{r}}=\mathrm{ae}^{\mathrm{b} T} \\
& \mathrm{Q}_{10}=\mathrm{e}^{10 \mathrm{~b}} \\
& C_{\mathrm{r}}=\mathrm{c} x+\mathrm{d}
\end{aligned}
$$

式中, $C_{\mathrm{r}}$ 表示沉积物有机碳矿化速率, $\mu \mathrm{mol} /\left(\mathrm{m}^{2} \cdot \mathrm{s}\right) ; T$ 为模拟柱中沉积物温度, ${ }^{\circ} \mathrm{C} ; x$ 为水位, $\mathrm{cm} ; \mathrm{a}$ 为基础碳 排放速率; $\mathrm{b}$ 为用于计算有机碳矿化温度敏感性系数的常数,由公式 (1) 获取; $\mathrm{c}$ 和 $\mathrm{d}$ 为常数.

采用 SPSS 软件(SPSS 20.0 for Windows) 进行数据分析. 采用单因素方差分析 (One-way ANOVA) 研究不 同水位条件下沉积物有机碳矿化速率差异, 采用 Pearson 相关分析研究沉积物有机碳矿化速率与温度的关 系,最小显著差异法 (LSD) 进行多重比较,显著水 平为 $\alpha<0.05$. 使用 Excel 2010 进行数据预处理,使 用 Origin 2017 绘图,研究区示意图使用 ArcGIS 10.2 软件绘制.

\section{2 结果与分析}

\section{1 不同水位条件下有机碳矿化特征}

水位变化改变了有机碳矿化速率及动态变化 (图 3), 试验开始前 $6 \mathrm{~d}$ 有机碳矿化速率振幅较大, 且 $\mathrm{T} 1 、 \mathrm{~T} 2$ 和 $\mathrm{T} 3$ 处理间不存在显著差异,随着试验 周期的延长,有机碳矿化速率呈下降趋势, $10 \mathrm{~d}$ 后 降幅减小且趋于平缓. 试验周期内, $\mathrm{T} 1$ 处理有机碳 矿化速率 $\left(0.44 \mu \mathrm{mol} /\left(\mathrm{m}^{2} \cdot \mathrm{s}\right)\right)$ 略低于 $\mathrm{T} 3$ 处理 $\left(0.46 \mu \mathrm{mol} /\left(\mathrm{m}^{2} \cdot \mathrm{s}\right)\right)$; 而 $\mathrm{T} 1$ 处理有机碳矿化速率 是 T2、T4 和 T5 的 $1.09 、 3.31$ 和 3.57 倍. 这说明水位

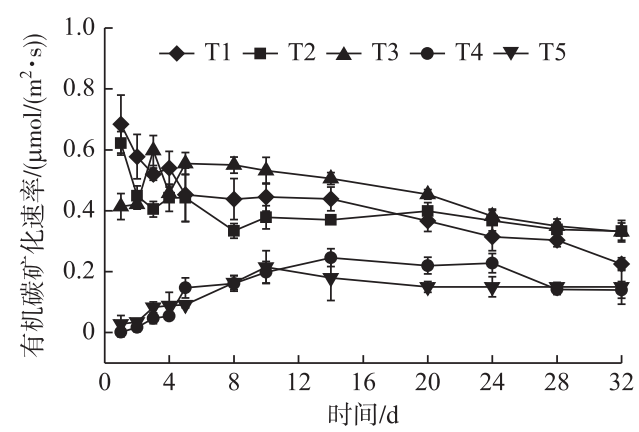

图 3 不同水位条件下干涸湖底沉积物有机碳排放特征 $(\mathrm{T} 1 、 \mathrm{~T} 2 、 \mathrm{~T} 3 、 \mathrm{~T} 4$ 和 T5 分别代表 $0 、-9 、-23 、-34$ 和 $-45 \mathrm{~cm}$ 水位处理; 数据均为均值土标准误,下同)

Fig.3 Mineralization rate of sediment organic carbon in the dry lake for different water level gradients 升高会促进有机碳矿化分解, 反之则抑制.

\section{2 不同水位条件下有机碳累积矿化量及动力学特征}

水位改变了沉积物 $\mathrm{CO}_{2}$ 累积矿化量 (图 4). 不同处理 $\mathrm{CO}_{2}$ 累积矿化量出现两个不同增长阶段, 即前期 ( $1 \sim 10 \mathrm{~d}$ ) 增长迅速和后期 ( $>10 \mathrm{~d}$ ) 增长缓慢. 其中 $\mathrm{T} 1$ 、 $\mathrm{T} 2$ 和 $\mathrm{T} 3$ 处理 $0 \sim 10 \mathrm{~d}$ 有机碳矿化速率显著高于 10 $32 \mathrm{~d}$, T4 和 T5 处理 $\mathrm{CO}_{2}$ 累积矿化量不存在显著性差异. $0 \sim 32 \mathrm{~d}$ 各处理沉积物有机碳累积矿化量表现为: T3 $\left(1.784 \mathrm{~g} / \mathrm{m}^{2}\right)>\mathrm{T} 1\left(1.718 \mathrm{~g} / \mathrm{m}^{2}\right)>\mathrm{T} 2\left(1.595 \mathrm{~g} / \mathrm{m}^{2}\right)>\mathrm{T} 4\left(0.510 \mathrm{~g} / \mathrm{m}^{2}\right)>\mathrm{T} 5\left(0.452 \mathrm{~g} / \mathrm{m}^{2}\right)$, 其中 $\mathrm{T} 1 、 \mathrm{~T} 2$ 和 $\mathrm{T} 3$ 处 


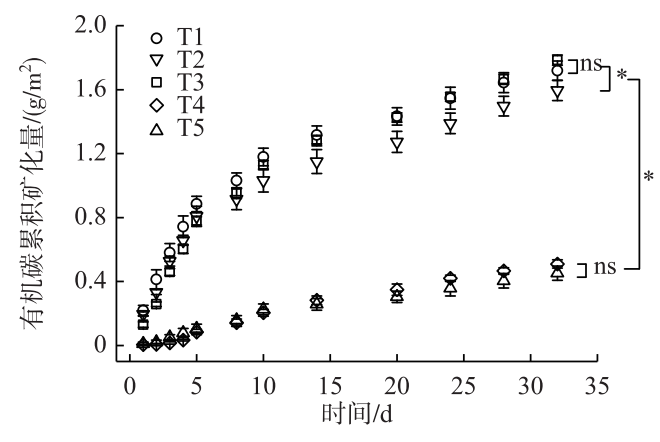

图 4 不同水位条件下干涸湖底 沉积物有机碳矿化量动态变化

Fig.4 Dynamic of cumulative mineralization of sediment organic carbon in the dry lake for different water level gradients

理显著高于 $\mathrm{T} 4 、 \mathrm{~T} 5$ 处理 $(P<0.05)$, 但 $\mathrm{T} 1$ 和 $\mathrm{T} 2 、 \mathrm{~T} 4$ 和 T5 处理间均无显著差异 (图 4). 有机碳累积矿化量 $\left(C_{t}\right)$ 占沉积物有机碳 $\left(C_{0}\right)$ 的比例 $\left(\mathrm{C}_{t} / \mathrm{C}_{0}\right)$ 介于 $(0.012 \sim$ 0.044 ) 之间 (表 3), T3 处理 $C_{t} / C_{0}$ 略高于 $\mathrm{T} 1$, 但二者间 不存在显著性差异, $\mathrm{T} 1$ 、 $\mathrm{T} 2$ 和 $\mathrm{T} 3$ 水位处理显著高于 $\mathrm{T} 4$ 和 $\mathrm{T} 5$ 处理.

一级反应动力学方程对不同水位处理 $\mathrm{CO}_{2}$ 潜在排 放量的模拟结果较好 (表 3). 干涸湖底沉积物 $\mathrm{CO}_{2}$ 潜在 排放量 $\left(C_{i}\right)$ 介于 $0.692 \sim 1.766 \mathrm{~g} / \mathrm{m}^{2}$ 之间, 其中 $\mathrm{T} 3$ 处理 $C_{i}$ 最高, $\mathrm{T} 5$ 处理最低; $\mathrm{T} 3$ 处理 $C_{i}$ 显著高于其他处理, $\mathrm{T} 1$ 和 $\mathrm{T} 2$ 处理以及 $\mathrm{T} 3$ 和 $\mathrm{T} 4$ 处理不存在显著性差异. $C_{i}$ 占 沉积物有机碳 $\left(C_{0}\right)$ 的比例 $\left(C_{i} / C_{0}\right)$ 介于 $0.018 \sim 0.045$ 之间. 与 $\mathrm{T} 1$ 处理相比, $\mathrm{T} 3$ 处理 $C_{i} / C_{0}$ 增加了 $8.2 \%, \mathrm{~T} 2$ 、 T4 和 T5 处理分别降低了 $8.6 \%$ 、46.3\% 和 $57.8 \%$. 水位 降低显著改变了有机碳矿化常数 $(\mathrm{k}), \mathrm{T} 1$ 处理 $\mathrm{k}$ 最高 $(0.137 \mathrm{~d}), \mathrm{T} 4$ 最低 $(0.032 \mathrm{~d})$. $\mathrm{T} 1$ 与 $\mathrm{T} 2$ 处理的 $\mathrm{k}$ 值差异不显著, 但 $\mathrm{T} 1$ 、 $\mathrm{T} 2$ 处理显著高于其他处理 $(P<0.05)$. 可知,水位变化改变了干涸湖底沉积物碳潜在释放量和周转速率.

表 3 不同水位处理干涸湖底沉积物有机碳矿化的一级动力学参数及 $C_{t} / C_{0} 、 C_{i} / C_{0}$ 值 *

Tab.3 Parameters of the first-order kinetics and $C_{t} / C_{0}, C_{i} / C_{0}$ values for

mineralization of sediment organic carbon in the dry lake for different water level gradients

\begin{tabular}{cccccc}
\hline 处理 & $C_{t} / C_{0}$ & $C_{i} /\left(\mathrm{g} / \mathrm{m}^{2}\right)$ & $C_{i} / C_{0}$ & $\mathrm{k} / \mathrm{d}$ & $R^{2}$ \\
\hline $\mathrm{T} 1$ & $0.044 \pm 0.002^{\mathrm{ab}}$ & $1.639 \pm 0.060^{\mathrm{ab}}$ & $0.042 \pm 0.002^{\mathrm{a}}$ & $0.137 \pm 0.013^{\mathrm{a}}$ & 0.986 \\
$\mathrm{~T} 2$ & $0.041 \pm 0.002^{\mathrm{b}}$ & $1.500 \pm 0.066^{\mathrm{b}}$ & $0.038 \pm 0.002^{\mathrm{b}}$ & $0.126 \pm 0.007^{\mathrm{a}}$ & 0.988 \\
$\mathrm{~T} 3$ & $0.046 \pm 0.001^{\mathrm{a}}$ & $1.776 \pm 0.043^{\mathrm{a}}$ & $0.045 \pm 0.001^{\mathrm{a}}$ & $0.098 \pm 0.006^{\mathrm{b}}$ & 0.990 \\
$\mathrm{~T} 4$ & $0.013 \pm 0.001^{\mathrm{c}}$ & $0.882 \pm 0.014^{\mathrm{c}}$ & $0.023 \pm 0.000^{\mathrm{c}}$ & $0.036 \pm 0.002^{\mathrm{c}}$ & 0.954 \\
$\mathrm{~T} 5$ & $0.012 \pm 0.001^{\mathrm{c}}$ & $0.692 \pm 0.021^{\mathrm{c}}$ & $0.018 \pm 0.001^{\mathrm{d}}$ & $0.032 \pm 0.010^{\mathrm{c}}$ & 0.979 \\
\hline
\end{tabular}

* 同列不同小写字母上标分别表示在 0.05 水平上存在显著差异.

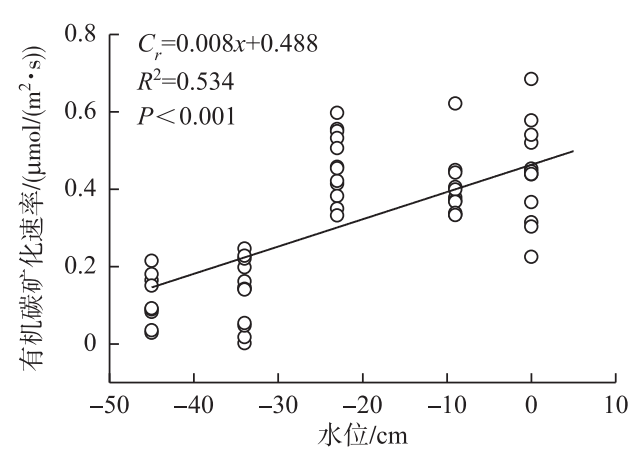

图 5 水位与沉积物有机碳矿化 速率的线性回归分析

Fig.5 Linear relationship between water level gradients and mineralization rate of sediment organic carbon in the day lake

\section{3 水位与沉积物有机碳矿化速率的相关性}

采用一次函数拟合干涸湖底沉积物有机碳矿化速率 与水位之间的关系 (图 5). 结果表明,一元线性方程能较 好地模拟水位与沉积物有机碳矿化的关系 $(P<0.01)$. 由 回归方程可知, 巴里坤湖干涸湖底沉积物水位每升高 1 $\mathrm{cm}$, 单位时间 $(\mathrm{s})$ 单位面积 $\left(1 \mathrm{~m}^{2}\right)$ 沉积物有机碳矿化量 将增加 $0.488 \mu \mathrm{mol}$.

\section{4 不同水位条件下有机碳矿化与温度的关系及温度敏 感性}

通过沉积物有机碳矿化速率与温度因子的相关分析 得出 (表 4), 不同水位条件下有机碳矿化速率均与温度 (ST) 显著相关, 其中 T5 处理有机碳矿化速率与沉积物温 度的相关性最高. 除 $\mathrm{T} 4$ 处理外, 其他处理有机碳矿化速 率与水体温度不存在显著相关性 $(P>0.05)$. Van't Hoff 指 数方程能较好地模拟不同水位处理下有机碳矿化速率与 沉积物温度的关系 $\left(R^{2}=0.396 \sim 0.547, P<0.001\right)$ (表 5 ). 
不同处理的 $Q_{10}$ 不同 (图 6), 表现为随水位的下降 $Q_{10}$ 升 高. 其中, $\mathrm{T} 1$ 处理 $\mathrm{Q}_{10}$ 值最低 (1.92), T5 处理最高 (2.92); T1、T2、T3 处理 $Q_{10}$ 不存在显著差异; $T 4$ 处理显 著高于 $\mathrm{T} 1$ 、 $\mathrm{T} 2$ 和 $\mathrm{T} 3$, 但低于 $\mathrm{T} 5$ 处理. 总体上,水位变化 改变了沉积物有机碳矿化温度敏感性, 即水位降低显著 提高了 $\mathrm{Q}_{10}$.

\section{3 讨论}

通过异位沉积物柱模拟湖泊干涸湖底沉积物有机 碳矿化对不同水位影响的研究表明,水位变化显著改变 了沉积物有机碳矿化动态变化过程和矿化速率. 水位变 化后 $0 \sim 10 \mathrm{~d}$ 内, T1、 T2 和 T3 处理的沉积物有机碳矿化 速率均呈下降趋势, T4 和 T5 处理则呈上升趋势, $10 \mathrm{~d}$ 后 均处于平缓状态且变幅相对较小. 水位对有机碳矿化速 率的影响主要表现在物理和生物两方面: (1) 水位变化 改变沉积物通气层厚度. $\mathrm{CO}_{2}$ 排放符合菲克定律, 扩散通 量与距离、温度以及氧气浓度等相关 ${ }^{[24-25]}$. 本研究沉积 物通气层厚度为 $0 \sim 45 \mathrm{~cm}$ 不等, -45 和 $-34 \mathrm{~cm}$ 处理由于 受含水量限制, 微生物对有机碳矿化量低, 且 $\mathrm{CO}_{2}$ 扩散距 离较长, 故表现为较低的 $\mathrm{CO}_{2}$ 通量. 而 -9 和 $-23 \mathrm{~cm}$ 水位 处理的 $\mathrm{CO}_{2}$ 累积排放量相对较高, 说明适当降低水位会 促进有机碳矿化, 且短距扩散会增加 $\mathrm{CO}_{2}$ 排放通量, 这与 杨钙仁等 ${ }^{[26]}$ 、汪浩等 ${ }^{[27]}$ 和 Chimner 等 ${ }^{[28]}$ 研究结果基本 一致; (2) 水位变化通过改变微生物活性进而影响有机 碳矿化速率. 研究表明, 当水位从淹水降至 $0 \mathrm{~cm}$ 以下时, $0 \sim 20 \mathrm{~cm}$ 沉积物微生物生物量碳显著增加 ${ }^{[29-31]}$, 本研究 中-9 $\mathrm{cm}$ 水位处理沉积物有机碳矿化速率显著高于 -34 和 $-45 \mathrm{~cm}$ 水位, 可能是因为水位变化改变微生物特征 从而影响有机碳矿化速率.

一级动力学方程拟合结果表明,水位对有机碳矿化 速率影响较大. 其中, $0 、-9$ 和 $-23 \mathrm{~cm}$ 水位处理 $C_{t} 、 C_{t}$ / $C_{0} 、 C_{i} 、 C_{i} / C_{0}$ 均显著高于 -34 和 $-45 \mathrm{~cm}$ 水位处理, 这与 其他研究结果大部分一致 ${ }^{[26,32-33]}$, 即水位降低增加沉积 物 $C_{t} 、 C_{i}$ 和 $k$ 值. $0 、-9$ 和 $-23 \mathrm{~cm}$ 水位处理 $C_{i} / C_{0}$ 和 $C_{t}$ / $C_{0}$ 相对较高, 表明其易分解有机碳 (可溶性有机碳) 高于 -34 和 $-45 \mathrm{~cm}$ 处理,这可能是因为低水位好氧条件下微 生物矿化作用受底物含量限制. 本研究表明, 沉积物有 机碳矿化速率 $\left(C_{\mathrm{r}}\right)$ 与水位 $(x)$ 呈显著正相关, 线性方程 $C_{\mathrm{r}}=0.008 x+0.488\left(R^{2}=0.534, P<0.001\right)$ 能很好地模拟 有机碳矿化速率与水位的关系. 但小叶章湿地的水位控 制试验研究表明, $\mathrm{CO}_{2}$ 排放量与水位呈显著负相关 $\left(C_{\mathrm{r}}=\right.$ $-31.29 x+926.92)^{[34]}$, 与本研究结果不一致, 这可能与植 被或沉积物基础性质存在差异有关。
表 4 干涸湖底沉积物有机碳矿化速率与 水体温度和沉积物温度的 Pearson 分析

Tab.4 Pearson correlation analysis between mineralization rate of sediment organic carbon and water temperature, sediment temperature in the dry lake

\begin{tabular}{ccc}
\hline 水位处理 & 沉积物温度 & 水体温度 \\
\hline T1 & $0.633^{*}$ & -0.067 \\
T2 & $0.571^{*}$ & 0.212 \\
T3 & $0.539^{*}$ & 0.266 \\
T4 & $0.750^{* *}$ & $0.571^{*}$ \\
T5 & $0.759^{* *}$ & 0.490
\end{tabular}

** 表示在 0.01 水平上显著相关, *表示在 0.05 水平 上显著相关 (双侧检验).

表 5 干涸湖底沉积物有机碳矿化 速率与温度的回归分析

Tab.5 Regression analysis between temperature and mineralization rate of sediment organic carbon in the dry lake

\begin{tabular}{cccc}
\hline 处理 & 回归方程 & $R^{2}$ & $P$ \\
\hline $\mathrm{T} 1$ & $C_{\mathrm{r}}=0.117 \exp (0.065 T)$ & 0.428 & $<0.001$ \\
$\mathrm{~T} 2$ & $C_{\mathrm{r}}=0.373 \exp (0.072 T)$ & 0.396 & $<0.001$ \\
$\mathrm{~T} 3$ & $C_{\mathrm{r}}=0.096 \exp (0.075 T)$ & 0.443 & $<0.001$ \\
$\mathrm{~T} 4$ & $C_{\mathrm{r}}=0.102 \exp (0.093 T)$ & 0.437 & $<0.001$ \\
$\mathrm{~T} 5$ & $C_{\mathrm{r}}=0.076 \exp (0.107 T)$ & 0.547 & $<0.001$ \\
\hline
\end{tabular}

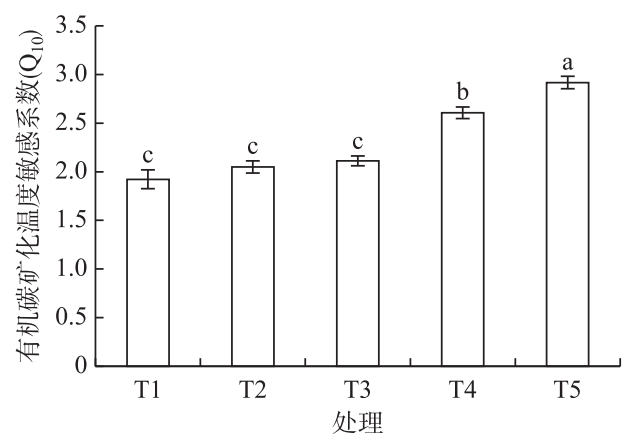

图 6 不同水位条件下干涸湖底沉积物 有机碳矿化温度敏感系数 $\left(Q_{10}\right)$

(不同小写字母表示 0.05 水平下 不同水位处理有机碳矿化温度敏感系数 间存在显著差异)

Fig.6 Temperature sensitivity of sediment organic mineralization $\left(Q_{10}\right)$ in the dry lake under different water level gradients 
温度调控微生物矿化有机碳的每个环节 ${ }^{[35]}$, 是影响 $\mathrm{CO}_{2}$ 排放的重要环境因子 ${ }^{[36-37]}$. 本研究中, 不同水位 处理有机碳矿化速率与沉积物 $5 \mathrm{~cm}$ 温度呈显著相关 $(P<0.05)$; 仅 $-34 \mathrm{~cm}$ 水位处理有机碳矿化速率与模拟 柱中水体温度显著相关. 研究表明, 温度对有机碳矿化的影响存在阈值, 即当温度低于某一阈值时 (约 $35^{\circ} \mathrm{C}$ ), 矿化速率随温度的上升而增加, 当温度超过此阈值时, 有机碳矿化受到抑 制 $^{[38]}$. 本研究中沉积物温 度 $\left(15 \sim 27^{\circ} \mathrm{C}\right)$ 均低于该阈值, 故有机碳矿化与温度呈显著的指数相关. $Q_{10}$ 受气候条件、植被以及人类活动 (放牧、施肥) 的影响 ${ }^{[39]}$, 干旱区盐湖干涸湖底沉积物 $Q_{10}$ 同样也受温度和水分影响. 相关研究表明, $Q_{10}$ 随水 位的降低呈增加趋势 ${ }^{[18]}$; 但也有研究表明水位升高同样会使 $Q_{10}$ 增加, 且 $Q_{10}$ 变化与微生物群落结构显著相 关 ${ }^{[40]}$, 与本研究结果一致, 这可能是由于水位变化改变了微生物对有机碳的矿化. 全球土壤 $Q_{10}$ 介于 $1.3 \sim$ 8.8 之间, 平均值为 $2.4^{[41]}$. $T 1 \sim T 5$ 处理的 $Q_{10}$ 分别为 $1.9 、 2.1 、 2.1 、 2.6$ 和 2.9 , 其中 $\mathrm{T} 1 、 \mathrm{~T} 2$ 和 $\mathrm{T} 3$ 水位处理的 $Q_{10}$ 低于全球土壤平均值, 而 $\mathrm{T} 4$ 和 $\mathrm{T} 5$ 的 $Q_{10}$ 显著高于全球平均值, 表明水位降低可能会提高 $Q_{10}$, 而气候变 暖可能会提高沉积物中有机碳的矿化速率, 从而对全球气候变化产生正反馈.

在未来气候变化和人为干扰仍可能加剧的背景下, 中国西北干旱区湖泊水位变化情况存在较多的不确 定性. 当湖泊水位处于持续下降情景下, 更多的有机碳暴露于空气, 将加快沉积物有机碳矿化速率. 本研究 表明干涸湖底沉积物水位从 $0 \mathrm{~cm}$ 下降至 -9 和 $-23 \mathrm{~cm}$ 时, 沉积物有机碳矿化速率呈升高趋势, 但低于全球 水体碳排放速率 $\left(1.92 \mathrm{~g} /\left(\mathrm{m}^{2} \cdot \mathrm{d}\right)\right)^{[8]}$. 而当水位下降至 -34 和 $-45 \mathrm{~cm}$ 时, 有机碳矿化速率显著低于 $0 、-9$ 和 $-23 \mathrm{~cm}$ 水位处理, 表明水位降至 $-23 \mathrm{~cm}$ 以下可能会抑制沉积物有机碳矿化. 水位下降同样也改变了 $Q_{10}$, 即随着水位下降有机碳矿化对温度的变化更为敏感. 因此, 湖泊水位降低可能会促进干旱区湖泊沉积物矿 化分解, 对全球气候变暖产生正反馈. 而当气候变暖冰川融雪增加时, 湖泊水位可能持续升高, 更多的沉积 物将处于厌氧环境. 本研究中水位从 $-45 \mathrm{~cm}$ 上升至 $0 \mathrm{~cm}$, 沉积物有机碳矿化速率呈先升后降趋势, $Q_{10}$ 则持 续下降, 这与胡保安等 ${ }^{\left[{ }^{[2]}\right]}$ 在巴音布鲁克天鹅湖的原位研究结果相同, 即水位升高后, 微生物矿化分解速率受 到抑制, 有机碳库的稳定性得到提高, 从而对全球气候变暖产生负反馈. 因此, 无论湖区水位如何变化, 沉积 物中有机碳矿化速率均会受到不同程度的影响. 在全球温度持续上升、降水增加以及人类活动干扰增强的 大背景下, 高盐湖泊沉积物中有机碳的稳定机制还有待进一步研究.

\section{4 结论}

不同水位处理沉积物有机碳矿化速率的动态变化不同, 水位下降有机碳矿化速率受抑制. 水位显著改 变了沉积物有机碳累积矿化量、有机碳潜在排放量占总有机碳的比和有机碳矿化常数. 水位与有机碳矿化 速率显著相关,水位每升高/降低 $1 \mathrm{~cm}$, 单位面积单位时间有机碳矿化量将增加/减少 $0.488 \mu \mathrm{mol}$. 有机碳矿 化速率与沉积物温度呈显著的指数函数关系, 且水位降低提升了有机碳矿化温度敏感系数. 气候变化和人 为干扰影响盐湖水位变化, 从而影响沉积物有机碳动态, 这可能是干旱区盐湖沉积物对气候变化的适应机 制, 以保障其有机碳库存及稳定性.

致谢: 感谢胡保安、王宁宁、韩东亮等在巴里坤湖采样过程中给予的帮助; 感谢陈蓓在 ArcGIS 绘图方面给予 的帮助.

\section{5 参考文献}

[ 1 ] IPCC. Climate change 2014: Synthesis report. contribution of working groups I , II and III to the fifth assessment report of the intergovernmental panel on climate change. Geneva: intergovernmental panel on climate change, 2014.

[ 2 ] Zhang YM, Hu CS, Zhang JB et al. Research advances on source/sink intensities and greenhouse effects of $\mathrm{CO}_{2}, \mathrm{CH}_{4}$ and $\mathrm{N}_{2} \mathrm{O}$ in agricultural soils. Chinese Journal of Eco-Agriculture, 2011, 19(4) : 966-975. DOI: 10.3724/SP.J.1011.2011. 00966. [张玉铭, 胡春胜, 张佳宝等. 农田土壤主要温室气体 $\left(\mathrm{CO}_{2} 、 \mathrm{CH}_{4} 、 \mathrm{~N}_{2} \mathrm{O}\right)$ 的源/汇强度及其温室效应研究进 展.中国生态农业学报, 2011, 19(4) : 966-975.

[ 3 ] Lehner B, Döll P. Development and validation of a global database of lakes, reservoirs and wetlands. Journal of Hydrolo$g y, 2004,296$ (1) : 1-22. DOI: 10.1016/j.jhydrol.2004.03.028.

[ 4 ] Allen GH, Pavelsky TM. Global extent of rivers and streams. Science, 2018, 361(6402) : 585-588. DOI: 10.1126/science.aat0636. 
[ 5 ] Messager ML, Lehner B, Grill G et al. Estimating the volume and age of water stored in global lakes using a geo-statistical approach. Nature Communications, 2016, 7: 13603. DOI: 10.1038/ncomms13603.

[ 6 ] Tranvik LJ, Downing JA, Cotner JB et al. Lakes and reservoirs as regulators of carbon cycling and climate. Limnology \& $O$ ceanography, 2009, 54(6) : 2298-2314. DOI: 10.4319/lo.2009.54.6_part_2.2298.

[ 7 ] Bastviken D, Tranvik LJ, Downing JA et al. Freshwater methane emissions offset the continental carbon sink. Science, 2011, 331(6013) : 50. DOI: 10.1126/science.1196808.

[ 8 ] Raymond PA, Hartmann J, Lauerwald R et al. Global carbon dioxide emissions from inland waters. Nature, $2013, \mathbf{5 0 3}$ (7476) : 355-359. DOI: 10.1002/2014GB004850.

[ 9 ] Cole JJ, Prairie YT, Caraco NF et al. Plumbing the global carbon cycle: integrating inland waters into the terrestrial carbon budget. Ecosystems, 2007, 10: 171-184. DOI: 10.1007/s10021-006-9013-8.

[10] Fierer N. Embracing the unknown: disentangling the complexities of the soil microbiome. Nature Reviews Microbiology, 2017, 15(10) : 579-590. DOI: 10.1038/nrmicro.2017.87.

[11] Gudasz C, Bastviken D, Steger K et al. Temperature-controlled organic carbon mineralization in lake sediments. Nature, 2010, 466(7305) : 478. DOI: 10.1038/nature09186.

[12] Marotta H, Pinho L, Gudasz C et al. Greenhouse gas production in low-latitude lake sediments responds strongly to warming. Nature Climate Change, 2014, 4( 6 ) :467-470. DOI: 10.1038/nclimate2222.

[13] Burdige DJ. Temperature dependence of organic matter remineralization in deeply-buried marine sediments. Earth \& Planetary Science Letters, 2011, 311(3) : 396-410. DOI: 10.1016/j.epsl.2011.09.043.

[14] Cardoso SJ, Enrich-Prast A, Pace ML et al. Do models of organic carbon mineralization extrapolate to warmer tropical sediments? Limnology \& Oceanography, 2014, 59(1) : 48-54. DOI: 10.4319/lo.2014.59.1.0048.

[15] Wurtsbaugh WA, Miller G, Null SE et al. Decline of the world's saline lakes. Nature Geoscience, 2017, 10: 816-821. DOI: $10.1038 /$ ngeo3052.

[16] Zhang X, Wu YH, Zhang X. Water level variation of inland lakes on the south-central Tibetan Plateau in 1972-2012. Acta Geographica Sinica, 2014, 69(7) : 993-1001. DOI: 10.11821/dlxb201407011. [张釒金, 吴艳红, 张金金. 1972-2012 年 青藏高原中南部内陆湖泊的水位变化. 地理学报, 2014, 69(7): 993-1001.]

[17] Ou Q, Wang JT, Zhou JH et al. Comparison of soil $\mathrm{CO}_{2}$ flux among different water levels in coastal wetlands. Chinese Journal of Applied and Environmental Biology, 2014, 20(6): 992-998. [欧强, 王江涛, 周剑虹等. 滨海湿地不同水位梯 度下的土壤 $\mathrm{CO}_{2}$ 通量比较. 应用与环境生物学报, 2014, 20(6):992-998.]

[18] Zhong QC, Guan YZ, Liu Q et al. Effects of water table manipulation on the soil respiration in a reclaimed tidal wetland at Dongtan of Chongming Island, China. Chinese Journal of Applied Ecology, 2013, 24(8): 2141-2150. [仲启铖, 关阅章, 刘倩等. 水位调控对崇明东滩围垦区滩涂湿地土壤呼吸的影响. 应用生态学报, 2013, 24(8): 2141-2150.]

[19] Hu BA, Jia HT, Zhu XP et al. Effect of water level on the soil respiration in a Swan Lake Wetland at Bayanbulak. Journal of Arid Land Resources and Environment, 2016, 30(7): 175-179. [胡保安, 贾宏涛, 朱新萍等. 水位对巴音布鲁克天 鹅湖高寒湿地土壤呼吸的影响. 干旱区资源与环境, 2016, 30(7): 175-179.]

[20] Wang SM, Dou HS eds. China lakes record. Beijing: Science Press, 1998: 354. [王苏民, 窦鸿身. 中国湖泊志. 北京: 科学出版社, 1998: 354.]

[21] Wang ZB, He QY, Yang SY et al. Comparison and application of Shepard's and Folk's classifications to the subsurface mapping in the south yellow sea. Marine Geology \& Quaternary Geology, 2008, 28(1)：1-8. [王中波, 何起祥, 杨守业 等. 谢帕德和福克碎屑沉积物分类方法在南黄海表层沉积物编图中的应用与比较. 海洋地质与第四纪地质, $2008,28(1): 1-8$. ]

[22] Bao SD ed. Soil agricultural chemistry analysis. Beijing: China Agricultural Science Press, 2000. [鲍士旦. 土壤农化分 析. 北京: 中国农业出版社, 2000.]

[23] Ma TE, Wei YC, Yang XL et al. Mineralization characteristics of soil organic carbon under long-term fertilization management. Chinese Journal of Eco-Agriculture, 2016, 24(1)：8-16. [马天娥, 魏艳春, 杨宪龙等. 长期施肥措施下土壤有 机碳矿化特征研究. 中国生态农业学报, 2016, 24(1):8-16.]

[24] Zeng YW ed. Fundamentals of inorganic materials science: Second edition. Wuhan: Wuhan University of Technology Press, 2015. [曾燕伟. 无机材料科学基础: 第 2 版. 武汉: 武汉理工大学出版社, 2015.]

[25] Mo DC, Xiao YL. Discuss the scope of the solution to Fick's second law. Chemistry, 1990, (6) : 50-51. [莫鼎成, 肖雅 龄. 关于菲克第二定律解的适用范围讨论. 化学通报, 1990, (6) : 50-51.] 
[26] Yang GR, Tong CL, Xiao AH et al. Effects of water content on redox potential and carbon mineralization of wetland sediments. Environmental Science, 2009, 30(8) : 2381-2386. DOI: 10.13227/j.hjkx.2009.08.049. [杨钲仁, 童成立, 肖和 艾等. 水分控制下的湿地沉积物氧化还原电位及其对有机碳矿化的影响. 环境科学, 2009, 30(8): 2381-2386.]

[27] Wang H, Yu LF, Chen LT et al. Responses of soil respiration to reduced water table and nitrogen addition in an alpine wetland on the Qinghai-Xizang Plateau. Chinese Journal of Plant Ecology, 2014, 38(6) : 619-625. DOI: 10.3724/SP.J. 1258.2014.00057. [汪浩, 于凌飞, 陈立同等. 青藏高原海北高寒湿地土壤呼吸对水位降低和氮添加的响应. 植物 生态学报, 2014, 38(6): 619-625.]

[28] Chimner RA, Pypker TG, Hribljan JA et al. Multi-decadal changes in water table levels alter peatland carbon cycling. Ecosystems, 2017, 20(5) : 1042-1057. DOI: s10021-016-0092-x.

[29] Yang GS, Song CC, Wang L et al. Influence of water level gradient on marsh soil microbial activity of Calamagrostis angustifoli. Environmental Science, 2010, 31(2) : 444-449. DOI: 10.13227/j.hjkx.2010.02.039. [杨桂生, 宋长春, 王丽 等. 水位梯度对小叶章湿地土壤微生物活性的影响. 环境科学, 2010, 31(2): 444-449.]

[30] Chimner RA, Cooper DJ. Influence of water table levels on $\mathrm{CO}_{2}$ emissions in a Colorado subalpine fen: an in situ microcosm study. Soil Biology \& Biochemistry, 2003, 35(3) : 345-351. DOI: 10.1016/S0038-0717( 02) 00284-5.

[31] Jaatinen K, Laiho R, Vuorenmaa A et al. Responses of aerobic microbial communities and soil respiration to water-level drawdown in a northern boreal fen. Environmental Microbiology, 2008, 10(2) : 339-353. DOI: 10.1111/j.1462-2920. 2007.01455.x.

[32] Zhang WJ, Tong CL, Yang GR et al. Effects of water on mineralization of organic carbon in sediment from wetlands. Acta Ecologica Sinica, 2005, 25(2): 249-253. [张文菊, 童成立, 杨钻仁等. 水分对湿地沉积物有机碳矿化的影响. 生 态学报, 2005, 25(2) : 249-253.]

[33] Ci E, Al-Kaisi MM, Wang LG et al. Soil organic carbon mineralization as affected by cyclical temperature fluctuations in a karst region of Southwestern China. Pedosphere, 2015, 25(4) : 512-523. DOI: 10.1007/s10021-016-0092-x.

[34] Hou CC. Effects of hydrological changes on soil carbon sequestration of marsh in the Sanjiang plain [Dissertation]. Changchun: Northeast Institute of Geography and Agricultural Ecology, Chinese Academy of Sciences, 2012. [侯翠翠. 水文条 件变化对三江平原沼泽湿地土壤碳蓄积的影响 [学位论文]. 长春: 中国科学院东北地理与农业生态研究 所, 2012.]

[35] Luo Y, Zhou X eds. Soil respiration and the environment. London: Elsevier, 2006: 85-92.

[36] Wang XY, Li YL, Zhao XY et al. Responses of soil respiration to different environment factors in semi-arid and arid Areas. Acta Ecologica Sinica, 2012, 32(15): 4890-4901. [王新源, 李玉霖, 赵学勇等. 干旱半干旱区不同环境因素对土壤 呼吸影响研究进展. 生态学报, 2012, 32(15): 4890-4901.]

[37] Wang HB, Ma MG, Wang XF et al. Carbon flux variation characteristics and its influencing factors in an alpine meadow ecosystem on eastern Qinghai-Tibetan plateau. Journal of Arid Land Resources and Environment. 2014, 28(6) : 50-56. [王 海波, 马明国, 王旭峰等. 青藏高原东缘高寒草甸生态系统碳通量变化特征及其影响因素. 干旱区资源与环境, $2014,28(6): 50-56$.

[38 ] Carey JC, Tang J, Templer PH et al. Temperature response of soil respiration largely unaltered with experimental warming. Proceedings of the National Academy of Sciences of the United States of America, 2016, 113(48) : 3797-3802. DOI: 10. 1073/pnas.1605365113.

[39] Peng SS, Piao SL, Tao W et al. Temperature sensitivity of soil respiration in different ecosystems in China. Soil Biology \& Biochemistry, 2009, 41(5) : 1008-1014. DOI: 10.1016/j.soilbio.2008.10.023.

[40] Ma'kiranta P, Laiho R, Fritze H et al. Indirect regulation of heterotrophic peat soil respiration by water level via microbial community structure and temperature sensitivity. Soil Biology \& Biochemistry, 2009, 41(4) : 695-703. DOI: 10.1016/j. soilbio.2009.01.004.

[41] Davidson EA, Verchot LV, Cattânio JH et al. Effects of soil water content on soil respiration in forests and cattle pastures of Eastern Amazonia. Biogeochemistry, 2000, 48(1) : 53-69. DOI: 10.1023/A:1006204113917.

[42] Hu BA, Jia HT, Zhu XP et al. Dynamics of soil respiration under different water conditions in an alpine wetland of the Xinjiang Bayanbulak Swan Lake. Research of Environmental Sciences, 2016, 29(7): 1041-1049. [胡保安, 贾宏涛, 朱 新萍等. 新疆巴音布鲁克天鹅湖高寒湿地土壤呼吸对水分条件的响应. 环境科学研究, 2016, 29(7): 1041-1049.] 